\title{
Riesgo de tsunami y planificación resiliente de la costa chilena. La localidad de Boca Sur, San Pedro de la Paz $\left(37^{\circ} \mathrm{S}\right)^{1}$
}

\author{
Carolina Martínez² y Rafael Aránguiz ${ }^{3}$
}

\begin{abstract}
RESUMEN
Se evalúa el riesgo de inundación por tsunami en la localidad de Boca Sur, comuna de San Pedro de La Paz $\left(37^{\circ} S\right)$, Región del Biobío. Se consideró un escenario extremo de tsunami generado por un sismo de magnitud Mw=9.0. La inundación por tsunami se obtuvo mediante modelación numérica usando el código NEOWAVE con 4 mallas anidadas de diferente resolución espacial y topo-batimetría de detalle. El análisis de vulnerabilidad consideró las dimensiones física, socioeconómica y organizacional, con datos obtenidos a través del Instituto Nacional de Estadística a nivel de manzana censal y encuestas a la población. Se determinó que el primer tren de ondas llega a la costa luego de 22 minutos de ocurrido el terremoto, alcanzando la cota de $5 \mathrm{msnm}$ y alturas de flujo de hasta $2 \mathrm{~m}$. Los factores de vulnerabilidad que explican el riesgo se asociaron a una alta precariedad de la vivienda, bajo nivel de bienestar social, alta densidad poblacional y bajo nivel de organización comunitaria de la población en caso de evacuación frente a tsunamis.
\end{abstract}

Palabras clave: Costa, desastres socionaturales, vulnerabilidad social, planificación Territorial

\begin{abstract}
We assessed the risk of flooding due to tsunamis in the Boca Sur neighborhood, located in the Municipality of San Pedro de la Paz $\left(37^{\circ} \mathrm{S}\right)$, Biobio region. We considered an extreme tsunami scenario generated by an earthquake of magnitude $\mathrm{Mw}$ 9.0. The tsunami inundation was obtained by means of the NEOWAVE model with 4 nested grids and detail topo-bathymetry data. The vulnerability analysis considered both physical, socioeconomical and organizational dimensions from statistical data given by the National Institute of Statistics (INE for its name in Spanish) at a block level as well as a field survey. Results show that the first tsunami waves would arrive 22 min post-earthquake and the maximum inundation reach up to 5 $\mathrm{m}$ above the mean water level with a flow depth of $2 \mathrm{~m}$. The risk level can be explained by several variables related to the vulnerability, namely, the prevalence of poor housing conditions, low level of social welfare, high population density and low level of community organization in case of necessary tsunami evacuation.
\end{abstract}

Key words: Coast, Natural Disasters, Vulnerability, Territorial Planning

\footnotetext{
Este trabajo ha sido realizado con apoyo de los proyectos FONDECYT No 1151367, 11140424 y FONDAP N¹5110017 de la Comisión Nacional de Investigación Científica y Tecnológica de Chile. Se agradece a la alumna tesista Constanza Villarroel (2012) por el levantamiento de datos de vulnerabilidad en Boca Sur, como parte de esta investigación. Artículo recibido el 30 de enero de 2016, aceptado el 26 de abril de 2016 y corregido el 16 de junio de 2016.
}

\footnotetext{
2 Instituto de Geografía, Pontificia Universidad Católica de Chile (Chile) / Centro Nacional de Investigación para la Gestión Integrada de Desastres Naturales, CONICYT/FONDAP/15110017 (Chile). E-mail: camartinezr@uc.cl

3 Departamento de Ingeniería Civil, Universidad Católica de la Santísima Concepción (Chile) / Centro Nacional de Investigación para la Gestión Integrada de Desastres Naturales, CONICYT/ FONDAP/15110017 (Chile).

E-mail: raranguiz@ucsc.cl
} 
Solo en la última década, seis tsunamis violentos y destructivos se generaron en la cuenca del Pacífico causando la muerte a más de 300.000 personas y una amplia devastación en los asentamientos humanos costeros de unos 17 países (Løvholt et al., 2014). Investigaciones asociadas a paleotsunamis han revelado una asociación entre ciclos sísmicos y grandes eventos lo cual ha incrementado la discusión sobre los Ilamados megatsunamis (Rhodes et al., 2011; Dura et al., 2015; Goff et al., 2014; Garret et al., 2015; Payande et al., 2015). Esto último genera la necesidad de evaluar el riesgo en áreas costeras afectadas por una recurrencia histórica importante e incorporar en la planificación territorial medidas de prevención y mitigación que permitan enfrentar los efectos de estos eventos, en especial porque el proceso de reconstrucción postdesastre suele ser lento, con un costo social y económico de gran envergadura que puede durar desde años a décadas.

Por ello, una parte importante de la investigación mundial ha estado centrada en evaluar los factores del riesgo que inducen y definen la magnitud del daño en términos de vidas humanas y pérdidas materiales. Asumiendo una tradición importante que define el riesgo natural como la plasmación territorial de una actuación humana poco acorde con las características físico-naturales del territorio donde tiene lugar (Blaikie et al., 1994; Rojas y Martínez, 2011), los factores principales están dados por la valoración de las condiciones asociadas a la amenaza (tsunami) y a la vulnerabilidad, que definen el riesgo. En el caso de la amenaza, la atención ha estado en determinar a través de modelamiento numérico, el comportamiento hidrodinámico del tsunami y su impacto en la infraestructura, especialmente a través de curvas de fragilidad (Suppasri et al., 2011; Mas et al., 2012; Sugawara \& Goto, 2012; Grilli et al., 2015; Prerna et al., 2015). Por su parte, la vulnerabilidad ha sido uno de los aspectos más discutidos por su difícil valoración en los procesos sociales y territoriales cambiantes. El énfasis ha estado en demostrar la sensibilidad o propensión al daño dentro del sistema socioecológico (Gallopín, 2006) o también enfocado a factores demográficos y socioeconómicos que incrementan o atenúan los impactos de una amenaza (Cutter et al., 2003).
Para ello, se han propuesto distintos modelos, entre los cuales destacan el de presión y liberación (Wisner et al., 2004), el modelo vulnerabilidad-sustentabilidad (Turner et al., 2003) y el modelo peligro del lugar (Cutter et al., 1996 y 2008). Actualmente, los elementos incluidos en la vulnerabilidad se relacionan con la exposición a una perturbación (estrés), la sensibilidad a dicha perturbación y su capacidad para adaptarse; por ello, pueden tratarse desde el enfoque de la adaptación al cambio climático (Adger, 2006; Angell \& Stokke, 2014; Yoo et al., 2014; Okey et al., 2015). Bajo el enfoque de la reducción del riesgo, esta última se vincula estrechamente con la resiliencia social, la que suele involucrar un proceso continuo de aprendizaje con el fin de que puedan tomarse las mejores decisiones en el manejo del riesgo (Cutter et al., 2008; Jin \& Lin, 2011).

En este sentido, la distinción entre vulnerabilidad social y resiliencia social se encuentra según algunos autores, en el proceso de transformación que sufre el sistema debido a la perturbación, donde la vulnerabilidad refleja el estado preimpacto y por ello define el daño, y la resiliencia se ubica en el proceso postimpacto y define la capacidad de recuperación o absorción del daño (Gallopín et al., 2006; Cutter et al., 2008; Shaw et al., 2014). En el caso del riesgo de tsunami, algunos modelos integran la exposición como factor independiente de la vulnerabilidad (Grezio et al., 2012). Otros a nivel teórico, incluyen la exposición y las capacidades de resistencia o robustez, las cuales en su conjunto explican el riesgo, sus daños directos e indirectos (Cochard et al., 2008). En general, la mayoría de las valoraciones del riesgo incluyen análisis probabilísticos, con apoyo de modelamiento numérico e integración de elementos de vulnerabilidad a algoritmos que sintetizan el riesgo (Kulikov et al., 2005; Geist \& Parsons, T., 2006; Løvholt et al., 2014a). Otros más recientes, incorporan el potencial de evacuación como vía para reducir el riesgo de desastre, a través de planes de evacuación incorporados en la valoración del riesgo (Ratna et al., 2010; Ratna, 2012, Tamburini, 2014; León \& March, 2014).

Considerando la alta recurrencia histórica que Chile presenta ante este tipo de amenazas (Lockridge, 1985; Lagos, 2000), sumado 
a los avances en el conocimiento de los ciclos sísmicos y su relación con el potencial de daño esperado, hace que se tengan suficientes herramientas técnico-científicas aplicables a la planificación territorial de la zona costera (Lagos y Gutiérrez, 2005; Lagos y Cisternas, 2008; Martínez et al., 2012). Otros factores menos conocidos, asociados a factores de vulnerabilidad y resiliencia social comenzaron a ser estudiados más sistemáticamente en el país a raíz de los efectos del terremoto y tsunami $\mathrm{Mw}=8.8 \mathrm{del} 27 / \mathrm{F}$ de 2010, sin embargo aún es una línea de investigación incipiente, frente a una necesidad cada vez más relevante, dada la intensa urbanización que se presenta en la costa del país (Martínez, 2014; Rojas et al., 2014; León \& March, 2014; Khew et al., 2015).

En este contexto, se evalúa el riesgo de inundación por tsunami en la localidad de Boca Sur en la comuna de San Pedro de la Paz, Área Metropolitana de Concepción. Esta localidad se ubica en el golfo de Arauco, en la denominada Ilanura de San Pedro, es decir una planicie litoral baja con desarrollo de antiguos pantanos, hoy urbanizados y convertidos a uso residencial. Por su cercanía a la costa y a la desembocadura del río Biobío, hace que por su localización geográfica presente una alta exposición al fenómeno de tsunami. La comuna de San Pedro de La Paz presenta un crecimiento urbano notable en las últimas décadas, pasando de 80.447 habitantes a 120.000 hab. en 10 años (INE, 2012), siendo la comuna con mayor crecimiento urbano $(2,41 \%)$ en el período 1996-2007 (Baeriswyl, 2009). Por ser un asentamiento caracterizado por bajos ingresos $(17,7 \%$ de la población en situación de pobreza) y de alta densidad poblacional, presenta condiciones de vulnerabilidad y riesgo no evaluadas frente a potenciales tsunamis. Esta área, aunque no fue afectada por el tsunami del 27/F de 2010, como otras localidades del Golfo de Arauco (Llico, Tubul y Arauco), es necesario evaluar si otros escenarios extremos pueden generar un daño potencial en la localidad para así aplicar medidas de mitigación adecuadas y reducir el riesgo de desastre, una preocupación central a nivel mundial e impulsada por Naciones Unidas a través del Marco de Acción de Sendai (2015-2030). En este sentido, la planificación para la resiliencia de la costa se entiende como una manera viable de integrar el conocimiento de las amenazas del lugar, las condiciones de vulnerabilidad y exposición a los mecanismos de preparación y la planificación territorial (expansión urbana, diseño urbano) que consideren el impacto de futuros desastres en la costa, previendo el daño potencial, reduciendo el tiempo de la emergencia y permitiendo que el sistema social o urbano siga funcionando. Esto último involucra rehabilitar o mitigar situaciones de riesgo en los asentamientos costeros ya existentes, o planificar los nuevos asentamientos considerando los peligros presentes así como fomentar la cohesión social y la participación comunitaria como estructura organizacional con capacidad de respuesta frente a la emergencia.

\section{Materiales y Métodos}

La localidad de Boca Sur se localiza en una extensa planicie litoral adyacente a la desembocadura del río Biobío $\left(37^{\circ} \mathrm{S}\right)$, denominada Llanura de San Pedro (Ilabaca, 1989). El origen de esta planicie se relaciona con procesos tectónicos y fluctuaciones del nivel del mar durante los últimos 6.000 años (Isla et al., 2012). Esto último posibilitó el desarroIlo de extensos humedales, playas y campos dunares, los cuales hoy en día se encuentran reducidos en superficie debido a las actividades antrópicas (Smith y Romero, 2009). El litoral presenta unos $18 \mathrm{~km}$ de largo y las alturas en la Ilanura no superan los $8 \mathrm{msnm}$. Inserta al interior del golfo de Arauco, esta zona se encuentra directamente expuesta a los efectos de tsunamis y aunque los antecedentes históricos en la región reportan la incidencia de unos 27 tsunamis de magnitud superior a $M=7$ entre 1562 y 2010, el área no habría sido afectada por grandes tsunamis al menos en épocas recientes (Palacios, 2012).

Desde el punto de vista administrativo, el área pertenece a la comuna de San Pedro de la Paz (Figura No 1). Está constituida por dos asentamientos: Boca Sur Nuevo y Boca Sur Viejo, el cual agrupa a unos 18.000 habitantes sobre una superficie de $3 \mathrm{~km}^{2}$. Ambos corresponden a barrios consolidados a partir de una política de erradicación de campamentos durante los años 80 y en la siguiente década, a programas de viviendas sociales. 
En general, el área se inserta en una comuna fundada en 1995 y que ha experimentado en las últimas décadas un explosivo crecimiento urbano por su rol residencial, ocupando el tercer lugar luego de Talcahuano y Concepción (Rojas et al., 2013).

Figura $\mathrm{N}^{0} 1$

Área de estudio

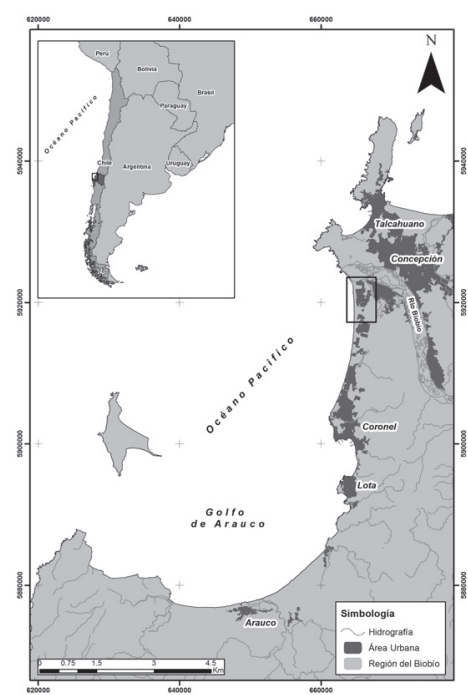

Fuente: Elaboración propia.

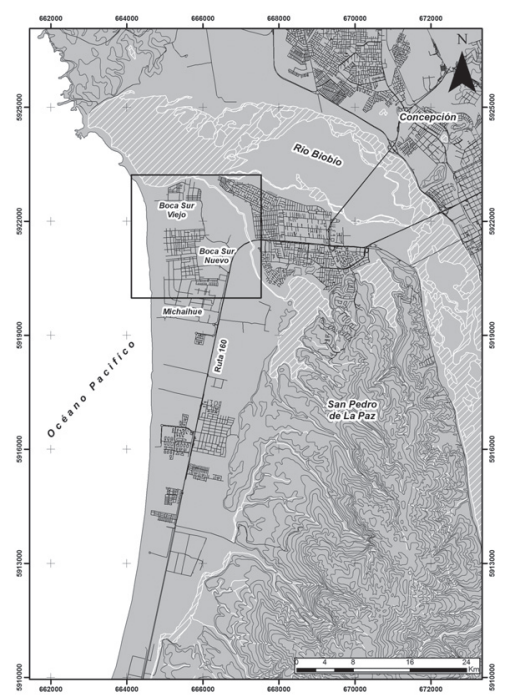

\section{Procedimientos}

Para la determinación de las áreas de riesgo de inundación por tsunami en la localidad se consideraron los criterios propuestos por Rojas y Martínez (2011) y Martínez (2013).

\section{Análisis de peligrosidad}

San Pedro de la Paz, ciudad vecina a Concepción, se emplaza en una zona sísmica que ha experimentado grandes eventos sísmicos y tsunamis tales como los ocurridos en 1570, 1657, 1730, 1751, 1835, 1960, 2010 (Aránguiz, 2010). Sin embargo, no hay registro histórico de inundación en San Pedro de la Paz o ingreso de agua por el río Biobío (Aránguiz \& Shibayama, 2013). La presencia del cañón submarino del Biobío y la isla Santa María juegan un rol fundamental en la propagación de tsunamis y efecto mitigador para San Pedro de la Paz, tal como quedó demostrado para el evento de febrero de 2010 (Aránguiz \& Shibayama, 2013). Es importante destacar, además, que el tsunami de 2010 ocurrió con marea baja, por lo tanto, la máxi-

ma inundación se vio reducida por este hecho (Aránguiz, 2010). Un estudio reciente analizó varios escenarios para la comuna de San Pedro de la Paz, esto es, tsunamis generados por sismos de magnitudes $M w=8.8,8.9$, y 9.0 (Kox et al., 2015). Se concluyó que un escenario desfavorable corresponde a un tsunami generado por un sismo de magnitud $\mathrm{Mw}=9.0$.

Por otro lado, después del Gran Terremoto y Tsunami del Este de Japón en marzo de 2011, los tsunamis pueden clasificarse en dos niveles según la frecuencia y propósito del análisis (Shibayama et al., 2013). El tsunami nivel 1 (con periodo de recurrencia de varias décadas hasta 100 años) corresponde a un evento frecuente que es utilizado para el diseño de estructuras de protección, mientras que el tsunami nivel 2 (con periodo de recurrencia de varios cientos o miles de años) es utilizado con fines de evacuación (Shibayama et al., 2013). Por lo tanto, analizando la recurrencia histórica de los eventos registrados en Chile Central, es posible definir un tsunami generado por un sismo de magnitud $\mathrm{Mw}=9.0$ como un tsunami nivel 2. Así, en el presente 
estudio se analizó un escenario de estas características agregando, además, un nivel de marea correspondiente a la máxima pleamar en sicigia de $1.2 \mathrm{msnm}$ (www.ioc-sealevelmonitorig.org) tal como lo recomienda la Guía para determinar la Inundación Potencial por Tsunami de Japón (MLIT, 2012).

Para estimar el área de inundación por tsunami, se utilizó modelación numérica mediante el modelo NEOWAVE (Yamazaki et al., 2009, 2011) con 4 mallas anidadas de diferente resolución espacial $\left(2^{\prime}, 30^{\prime \prime}, 6^{\prime \prime}\right.$ y $\left.1^{\prime \prime}\right)$. Las mallas de simulación se construyeron a partir de datos GEBCO, Cartas Náuticas, batimetrías de detalle de la zona de estudio y topografía LIDAR de $2.5 \mathrm{~m}$ de resolución. Las mallas utilizadas se muestran en la Figura $\mathrm{N}^{\circ} 2$. El recuadro en la malla 1 corresponde a los 4 segmentos utilizados para construir la condición inicial de tsunami. La longitud total de ruptura y el ancho se calcularon mediante las ecuaciones de Papazachos et al., (2004) dadas por las expresiones (1) y (2) en función de la magnitud del evento sísmico, obteniéndose un largo y ancho de $L=600 \mathrm{~km}$ y $W=150 \mathrm{~km}$, respectivamente. Ambas ecuaciones han demostrado dar buenos resultados para sismos en la zona de subducción chilena (Aránguiz et al., 2014). El desplazamiento interplaca se obtuvo a partir de la ecuación de momento sísmico dado por la expresión (3), donde $L$ es la longitud de ruptura, $W$ es el ancho, $D$ es el desplazamiento interplaca, $\mu$ una constante que vale $3 \times 10^{\wedge} 10 \mathrm{~Pa}$ y Mo es el momento sísmico calculado como $4.05 \times 10^{\wedge} 22 \mathrm{Nm}$ equivalente a una magnitud 9.0 según la expresión (4). Dada la configuración de la zona de subducción, se definieron 4 segmentos de $150 \mathrm{~km}$ de longitud cada uno. Los parámetros sísmicos de cada segmento se muestran en el Cuadro $\mathrm{N}^{\circ} 1$.

$$
\begin{aligned}
& \log (L)=0.55 M_{w}-2.19 \\
& \log (W)=0.31 M_{w}-0.63 \\
& M_{O}=\mu \operatorname{LWD} \\
& M_{w}=2 / 3 \log \left(M_{O}-9.1\right)
\end{aligned}
$$

A partir de los resultados del modelamiento numérico, se elaboró una carta de peligro de inundación por tsunami para la localidad de San Pedro de la Paz utilizando SIG ArcGis 10.3, en la cual se incluye el área de inundación máxima asociada al tsunami nivel 2. Se definieron tres niveles de peligrosidad, es decir profundidades de flujo menor a $0.5 \mathrm{~m}$, entre 0.5 y $2.0 \mathrm{~m}$ y mayor a $2.0 \mathrm{~m}$. Las áreas con inundación menor a $0.5 \mathrm{~m}$ consideradas como de peligro bajo, se extendieron hasta la calle Daniel Belmar (límite de la manzana censal), para así integrar la totalidad del área de estudio a la síntesis del riesgo.

\section{Cuadro $\mathrm{N}^{\circ} 1$}

Parámetros sísmicos de los cuatro segmentos que constituyen la fuente del tsunami para un evento de magnitud $M_{w} 9.0$

\begin{tabular}{|l|c|r|r|r|r|r|r|}
\hline Segmento & X0 & Y0 & $\begin{array}{r}\text { Profundidad } \\
(\mathrm{km})\end{array}$ & $\begin{array}{r}\text { Dislocación } \\
\left(^{\circ}\right)\end{array}$ & Rumbo & $\begin{array}{c}\text { Buzamiento } \\
\left(^{\circ}\right)\end{array}$ & $\begin{array}{c}\text { Deslizamiento } \\
\left(^{\circ}\right)\end{array}$ \\
\hline S1 & -74.9529 & -38.4813 & 30 & 15 & 10 & 18 & 111 \\
S2 & -74.647 & -37.1534 & 30 & 15 & 18 & 18 & 119 \\
S3 & -74.363 & -35.877 & 30 & 15 & 22 & 18 & 123 \\
S4 & -73.4942 & -34.5854 & 30 & 15 & 22 & 18 & 123 \\
\hline
\end{tabular}

Fuente: Elaboración propia. 
Figura $\mathrm{N}^{\circ} 2$

Mallas de simulación numérica

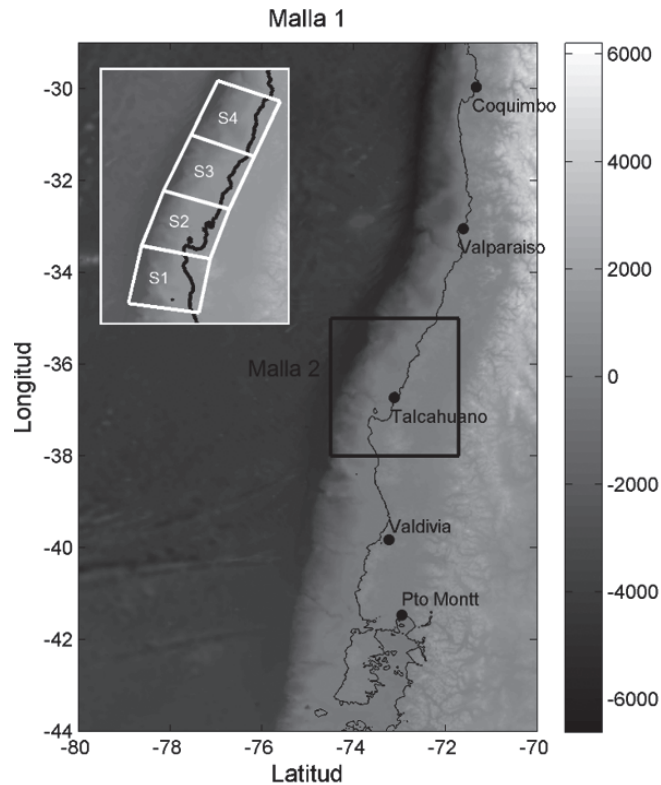

Fuente: Elaboración propia.

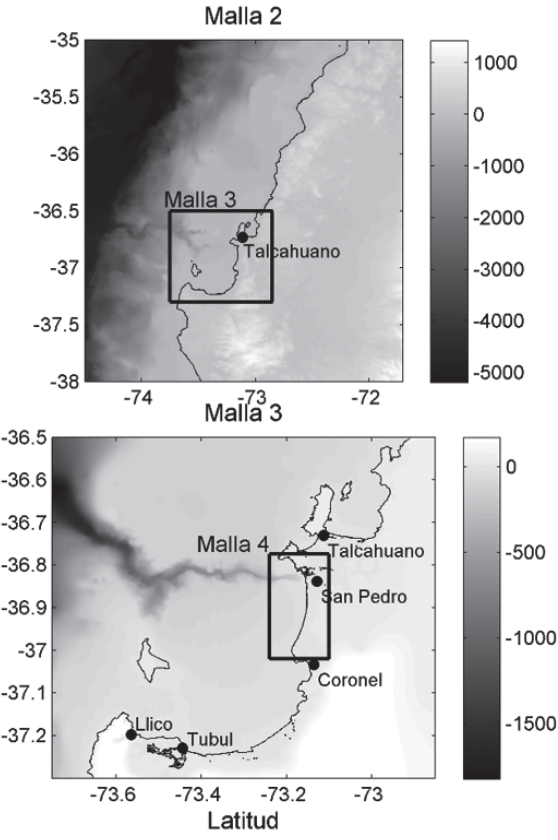

Cuadro $\mathrm{N}^{\circ} 2$

Niveles de peligrosidad para profundidad de flujo

\begin{tabular}{|l|l|l|}
\hline \multicolumn{2}{|l|}{ Profundidad de inundación } & \\
\hline Rango & Descriptor & Nivel de Peligro \\
\hline $0-0.5 \mathrm{~m}$ & Altura de las rodillas o menos & Bajo \\
\hline $0.5-2 \mathrm{~m}$ & Sobre la rodilla y más arriba de la cabeza & Medio \\
\hline$>2 \mathrm{~m}$ & $\begin{array}{l}\text { Mucho más arriba de la cabeza de una } \\
\text { persona }\end{array}$ & Alto \\
\hline
\end{tabular}

Fuente: modificado de Walsh et al., 2005.

\section{Análisis de vulnerabilidad}

La vulnerabilidad social aquí es entendida como la capacidad inherente de un sistema para soportar impactos adversos, provenientes de múltiples estresores a los cuales está expuesto, generando un daño potencial (Yang et al., 2015). Se consideraron tres tipos de vulnerabilidad específica (física, socioeconómica y organizacional), definidas según los criterios de Wilches-Chaux (1993). Para cada una de estas, se incluyeron variables representativas indicadas en el Cuadro $N^{\circ} 3$. Los datos para la dimensión física y socioeconómica fueron obtenidos del Censo 2002 (INE, 2002) a nivel de manzana censal y extraídas a través del software Redatam. Para la dimensión organizacional, se aplicaron encuestas a la población. Para ello, se aplicó un muestreo estratificado con confiabilidad de $90 \%$ y una precisión del 5\%. Considerando un universo de 10.093 personas, se determinó una muestra de 388 
encuestas, las cuales se aplicaron a personas entre 15 y 59 años.

Los datos fueron automatizados en plataforma SIG, para la obtención de mapas de síntesis que establecieron tres niveles de vulnera- bilidad (alto, medio y bajo) definidos a través de criterios de ponderación (Cuadro $\mathrm{N}^{\circ} 5$ ). Los niveles de vulnerabilidad se obtuvieron a través de álgebra de mapas en plataforma SIC según los criterios de Martínez et al. (2012).

\section{Cuadro $\mathrm{N}^{\circ} 3$}

Variables asociadas a cada dimensión de la vulnerabilidad

\begin{tabular}{|l|l|l|}
\hline Dimensión Física & Dimensión Socioeconómica & Dimensión Organizacional \\
\hline Tipo de vivienda & Densidad de población & $\begin{array}{l}\text { Organizaciones sociales vincu- } \\
\text { ladas a la gestión del riesgo }\end{array}$ \\
\hline Materialidad de la vivienda & Nivel de Hacinamiento & Sistema de alarma de tsunami \\
\hline & $\begin{array}{l}\text { Índice de Bienestar social } \\
\text { (IBS) }\end{array}$ & Reacción ante el evento \\
\hline
\end{tabular}

Fuente: Elaboración propia.

\section{Cuadro $\mathrm{N}^{\circ} 4$}

Niveles de vulnerabilidad asignados según variable

\begin{tabular}{|l|c|l|l|}
\hline Variable & \multicolumn{1}{|c|}{ Vulnerabilidad Alta } & Vulnerabilidad Media & \multicolumn{1}{|c|}{ Vulnerabilidad Baja } \\
\hline Tipo de vivienda & $\begin{array}{l}\text { Mediagua, conventillo } \\
\text { o choza }\end{array}$ & Casa o departamento & $\begin{array}{l}\text { Edificios de más de 3 } \\
\text { pisos }\end{array}$ \\
\hline $\begin{array}{l}\text { Materialidad de la } \\
\text { vivienda }\end{array}$ & $\begin{array}{l}\text { Adobe, piedra o ma- } \\
\text { dera }\end{array}$ & Concreto o madera & Hormigón, albañilería \\
\hline $\begin{array}{l}\text { Número de vivien- } \\
\text { das }\end{array}$ & ---- & --- \\
\hline $\begin{array}{l}\text { Densidad de pobla- } \\
\text { ción (hab./km²) }\end{array}$ & $249,77-681,07$ & $50,1-249,76$ & $0,50-0,53$ \\
\hline $\begin{array}{l}\text { Nivel de hacina- } \\
\text { miento }\end{array}$ & $50,1-75$ & $15,1-50$ & $0-15$ \\
\hline $\begin{array}{l}\text { Índice de Bienestar } \\
\text { Social (IBS)* }\end{array}$ & $15,2-100$ & $7,8-15,1$ & $0-7,7$ \\
\hline $\begin{array}{l}\text { Organizaciones so- } \\
\text { ciales }\end{array}$ & Reconoce instituciones & $\begin{array}{l}\text { Reconoce pero estas } \\
\text { son poco confiables }\end{array}$ & $\begin{array}{l}\text { No reconoce institu- } \\
\text { ciones }\end{array}$ \\
\hline $\begin{array}{l}\text { Sistema de alarma } \\
\text { de tsunami }\end{array}$ & Reconoce instituciones & $\begin{array}{l}\text { Reconoce pero estas } \\
\text { son poco confiables }\end{array}$ & $\begin{array}{l}\text { No reconoce institu- } \\
\text { ciones }\end{array}$ \\
\hline $\begin{array}{l}\text { Reacción ante el } \\
\text { evento }\end{array}$ & Inadecuada & Adecuada & Optima \\
\hline
\end{tabular}

*IBS: porcentaje de pobreza por manzana (MIDEPLAN, 2002)

Fuente: Elaboración propia. 
3. Riesgo de inundación por tsunami

La síntesis del riesgo se realizó mediante álgebra de mapas utilizando plataforma SIG y de acuerdo con los criterios de Eckert et al. (2012) y Jalínek et al. (2012) en Martínez et al. (2012), obteniéndose tres niveles de riesgo: alto, medio y bajo con puntajes desde 1 a 9 (Cuadro $N^{\circ}$ 5). Se incluyeron en esta síntesis, las vías de evacuación establecidas por la Ilustre Munici- palidad de San Pedro de la Paz (http://sanpedrodelapaz.cl/), con el fin de contextualizar la situación de riesgo de la población frente al potencial de evacuación en el caso de que un evento de estas características afecte el área, utilizándose para ello los criterios expuestos en Tamburini (2014), donde se establecen distintas velocidades de escape en función de las características físicas y etarias de la población evacuada.

Cuadro $\mathrm{N}^{\circ} 5$

Matriz de riesgo de inundación por tsunami, localidad de Boca Sur

\begin{tabular}{|c|l|r|r|r|}
\hline \multicolumn{2}{|c|}{ X } & \multicolumn{3}{|c|}{ Peligro } \\
\hline \multirow{3}{*}{ Vulnerabilidad } & \multicolumn{1}{|c|}{ Nivel } & Bajo (1) & Medio (2) & Alto (3) \\
\cline { 2 - 5 } & Bajo (1) & B $1 \times 1=1$ & B $1 \times 2=1$ & M $1 \times 3=3$ \\
\cline { 2 - 5 } & Medio (2) & B $2 \times 1=2$ & M $2 \times 2=4$ & A $2 \times 3=6$ \\
\cline { 2 - 6 } & Alto (3) & M $3 \times 1=3$ & A $3 \times 2=6$ & A $3 \times 3=9$ \\
\hline
\end{tabular}

Rango de riesgo: Bajo (1-2), Medio (3-4), Alto (6-9) Fuente: Martínez et al. (2012).

\section{Peligro, vulnerabilidad y áreas de riesgo de inundación por tsunami en la localidad de Boca Sur}

La Figura $N^{\circ} 3$ muestra los resultados de la modelación numérica mediante secuencia de imágenes de la propagación. El tiempo t=2 min corresponde al inicio del tsunami, esto es una vez terminado el sismo que se asumió con una duración de 2 min. Se observa que el tsunami ingresa al golfo de Arauco a los 12 min de iniciado el terremoto. A los 18 y 22 min se observan los fenómenos de refracción y difracción que sufre el frente principal producto de la presencia del cañón submarino del Biobío y la isla Santa María (Aránguiz \& Shibayama, 2013). De este modo, la primera onda del tsunami alcanzaría la costa de San Pedro de la Paz y desembocadura del río Biobío en 22-25 min.

Figura $\mathrm{N}^{\circ} 3$

Secuencia de propagación del tsunami desde la zona de generación al golfo de Arauco
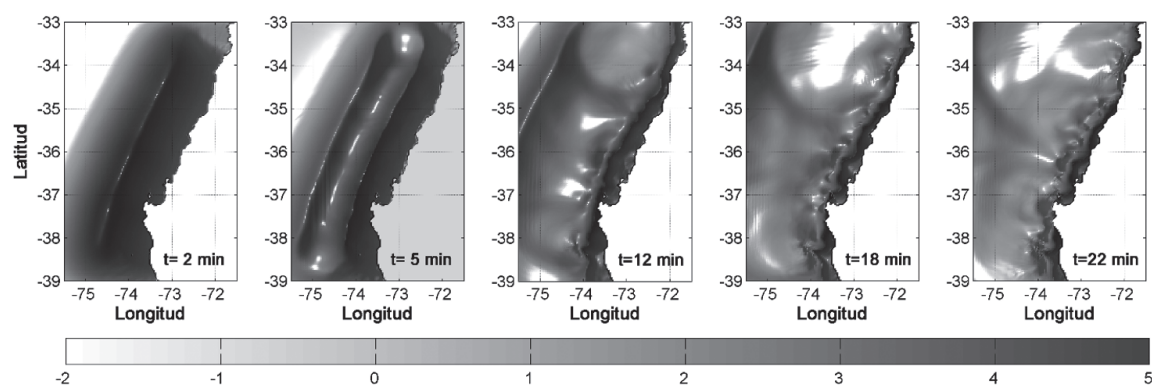

1

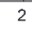

Fuente: Elaboración propia. 
La Figura $N^{\circ} 4$ muestra el comportamiento del tsunami en la zona de Boca Sur. La Figura 4-a muestra la variación del nivel del mar frente a la zona de estudio. Se observa que la primera onda comienza su arribo inmediatamente después del sismo, lo que concuerda con lo observado en el reciente tsunami de Illapel en septiembre de 2015 (Aránguiz et al., 2015) dado que la zona de estudio está sobre la zona de generación del tsunami. Sin embargo, la máxima amplitud se observa a los 25 min después de iniciado el sismo debido principalmente al ancho y profundidad de la plataforma continental en la zona del Biobío, a diferencia de lo observado en la zona de Coquimbo (Aránguiz et al., 2015).

Es importante destacar que la máxima amplitud se produce para la primera onda del tsunami, lo que implica un escenario desfavorable en términos de tiempo de evacuación. La Figura $N^{\circ}$ 4-b muestra el área de inundación en términos de la profundidad de flujo. En general se observa que el tsunami ingresa tanto por el río Biobío como por el humedal Los Batros, cruzando el eje de la Av. Pedro Aguirre Cerda. Se puede observar que el tsunami sobrepasa el sistema dunario y la inundación alcanza hasta la calle 12 de Febrero, esto es, una distancia horizontal de $1.200 \mathrm{~m}$. El sector más afectado corresponde a Boca Sur Viejo, donde el agua ingresa tanto por la costa como por el río. A pesar de la gran área de inundación, las profundidades de flujo no superan los $2 \mathrm{~m}$ en los sectores urbanizados. Las áreas con peligro alto, corresponden a una extensa zona litoral que cubre desde la playa hasta una distancia entre $800 \mathrm{~m}$ y 1,2 $\mathrm{km}$ al interior, siendo el sector más afectado Boca Sur Viejo, donde la inundación sobrepasa las primeras líneas de edificación, afectando principalmente viviendas sociales (Figura $\left.N^{\circ} 4-b\right)$.

Otra parte importante del área es afectada por peligro medio, alcanzando la cota de 5 msnm en Boca Sur Nuevo. Las áreas con peligro medio cubren la mitad de los asentamientos humanos en Boca Sur Nuevo y casi la totalidad en Boca Sur Viejo. En el caso del estero los Batros, la inundación alcanza la cota de los $5 \mathrm{msnm}$. Entre Boca Sur Viejo y Nuevo, el cordón dunar debido a su mayor altura, disminuye la capacidad de inundación del tsunami, generando un peligro bajo, lo mismo ocurre en otras áreas de dunas remanentes cercanas a la desembocadura.

Figura $\mathrm{N}^{\circ} 4$

Mareograma sintético frente a Boca Sur (a) y profundidad de flujo en la zona de estudio (b). $M$ indica la ubicación del mareógrafo sintético

(a)

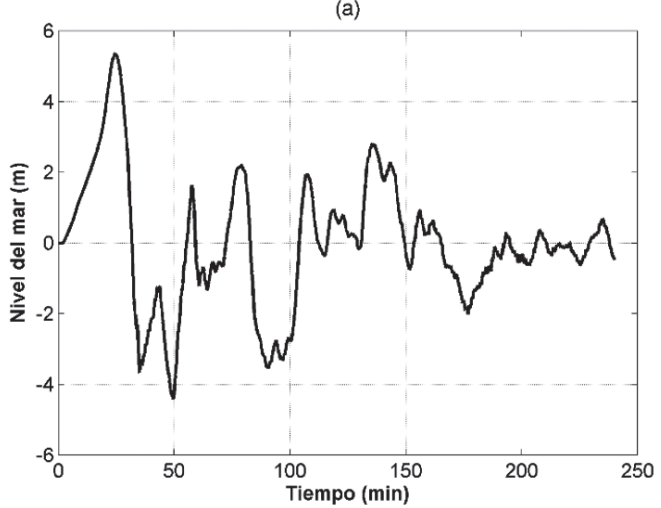

(b)

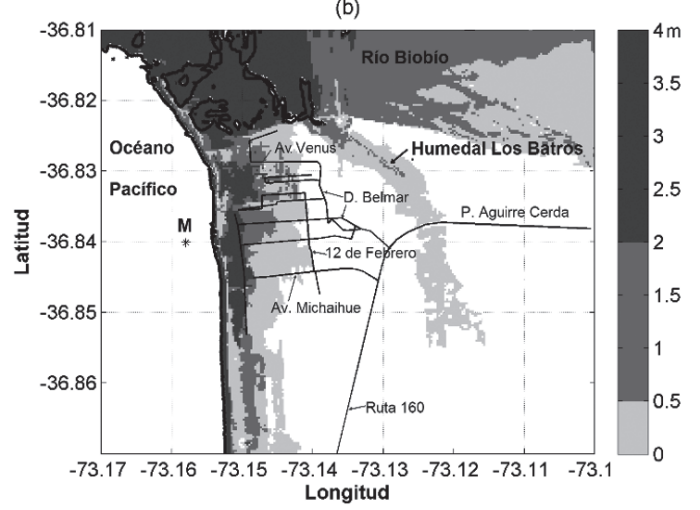

Fuente: Elaboración propia. 
Figura $\mathrm{N}^{\circ} 5$

Áreas de peligro de inundación por tsunami, localidad de Boca Sur

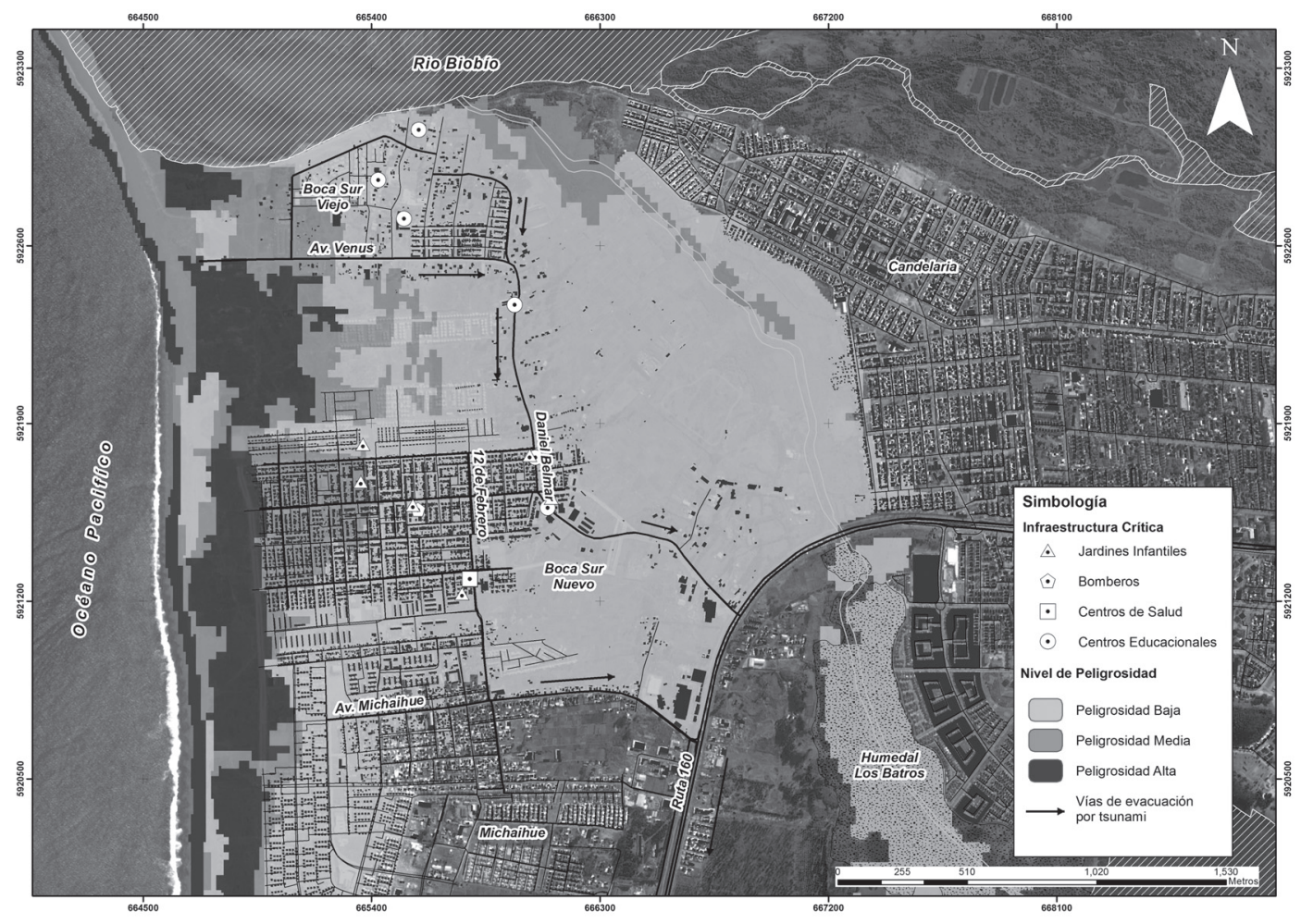

Fuente: Elaboración propia.

\section{Vulnerabilidad por tsunami en la localidad de Boca Sur}

La vulnerabilidad física en Boca Sur Viejo en función del tipo de vivienda, estableció vulnerabilidad alta y media debido al predominio de viviendas sociales de tipo casa o departamento sobre un uso de suelo residencial. Las viviendas más precarias (mediagua y choza) se localizaron cerca de la playa y a orillas del río Biobío en Boca Sur Viejo, la mayoría pequeñas, antiguas y construidas de materiales ligeros. Estas viviendas se vinculan a antiguas poblaciones de pescadores (Figura $\mathrm{N}^{\circ}$ 6A). Dado que en el área, especialmente en Boca Sur Nuevo predominaron las viviendas sociales construidas en albañilería, madera o mixtas, la vulnerabilidad se registró como media (Figura $N^{\circ} 7$ ). En general, el 7\% de la superficie del área presentó vulnerabilidad alta, mientras que el $93 \%$ restante registró nivel medio, involucrando a unas 16.000 personas (85\% de la población total).

La vulnerabilidad socioeconómica se registró como alta en el 16\% del área (Figura $\mathrm{N}^{\circ} 8$ ), mientras que la vulnerabilidad media ocupó una superficie de $84 \%$ del total, involucrando al $61 \%$ de la población $(11.000$ habitantes). La densidad de población fue variable según localidad: baja en Boca Sur Viejo y alta en Boca Sur Nuevo. Lo anterior se debió a que en la segunda predominaron las viviendas de pocos metros cuadrados de superficie, característico de las viviendas sociales (tipo bloques). Aquí la población involucrada con vulnerabilidad alta fue de 12.000 habitantes $(75 \%$ del total) versus 694 en Boca Sur Viejo (14\% del total). Desde el punto de vista del hacinamiento, Boca Sur Viejo presentó mayor hacinamiento que Boca Sur Nuevo, sin embargo predominó el hacinamiento medio en ambos sectores. 
El Índice de Bienestar Social (IBS) presentó alta vulnerabilidad, dado que en ambas localidades los valores fueron superiores al promedio nacional $(15,1 \%)$, siendo mayores en Boca Sur Viejo. En Boca Sur Nuevo, pequeños sectores muy dispersos (algunas manzanas censales) presentaron vulnerabilidad media, aquí el IBS estuvo entre $7 \%$ y $15 \%$. En síntesis, la pobreza aquí se registró para el $93 \%$ del área, elevando la vulnerabilidad y solo en un $7 \%$ se registró como media.

Desde el punto de vista organizacional, en Boca Sur Nuevo, el 35\% del total de encuestados reconoció no saber cuáles son las instituciones ligadas al riesgo de tsunami, $46 \%$ las reconoce pero no confía en ellas. El $19 \%$ reconoce las instituciones y participa de ellas, siendo las más nombradas Carabineros y Bomberos. En cambio en Boca Sur Viejo, el $63 \%$ de los encuestados indicó no reconocer instituciones ligadas al riesgo de tsunami; el $16 \%$ reconoció instituciones ligadas al riesgo de tsunami pero la cataloga de poco confiables y el $21 \%$ reconoció instituciones ligadas al riesgo de tsunami siendo las más confiables Bomberos y ONEMI.

Respecto al conocimiento sobre instituciones encargadas del sistema de alarma de tsunami en el país, en Boca Sur Viejo el $32 \%$ de los encuestados no reconoció organismos encargados de los sistemas de alarmas, 19\% reconoce saber cuáles son las instituciones ligadas a los sistemas de alarma pero no confía en ellas, $49 \%$ de la población sabe cuáles son los organismos encargados, producto del terremoto de 2010. En Boca Sur Nuevo, el $11 \%$ no conoce cuáles son las instituciones ligadas al sistema de alarma de tsunami; el $28 \%$ las conoce pero no confía en ellas (muchos dieron de ejemplo al SHOA y ONEMI), mientras que el $61 \%$ de la población conoce las instituciones ligadas al sistema de alarma.

De ahí que la vulnerabilidad organizacional fue media en ambos sectores (Figura $\mathrm{N}^{\circ}$ 9). Si bien, hubo graves problemas de conocimientos del término "tsunami", en cuanto a su origen y significado, la población está consciente que ante un evento fuerte como el del pasado 27/F de 2010, ellos deben evacuar de manera preventiva.
Sus reacciones frente al evento fueron variadas, puesto que las personas de mayor edad manifestaron no dejar sus casas por miedo al robo de sus pertenencias, otras personas sin saber muy bien cuáles eran las zonas seguras dejaron sus hogares y caminaron a la ruta 160 , y un pequeño número de habitantes había escuchado hablar de zonas seguras dentro de su comuna y se dirigieron hacia ellas.

Después del 27 de febrero de 2010, por parte de las autoridades hubo un interés en generar conciencia y conocimiento sobre una Plan de Evacuación, sin embargo este esfuerzo no prosperó. Si bien la Municipalidad de San Pedro de la Paz generó un mapa de zonas seguras y vías de evacuación, la población del barrio Boca Sur no lo conoce, incluso mostrándoles el mapa ellos manifestaban no conocerlo. Por las calles se divisa señalética de evacuación de tsunamis, y como la entrada a este barrio es solo una calle (Av. Daniel Belmar) es lógica la vía de evacuación a la ruta 160 .

Durante el trabajo de terreno se destacó una especial preocupación por un Plan de Evacuación por parte de los colegios, puesto que ellos deben velar por la correcta evacuación de cientos de niños de manera organizada y al no tener información esto se vuelve confuso y preocupante, ya que en la zona de Boca Sur Viejo hay un colegio con alumnos discapacitados en sillas de ruedas y con deficiencias mentales, en esa zona el camino es de ripio y evacuación con sillas de ruedas no es viable.

En Boca Sur, 25\% del total de la población reaccionó de manera inadecuada, es decir no evacuó el área; el 69\% de la población reaccionó de manera adecuada, si bien evacuaron, no sabían con certeza el área de seguridad y solo un 6\% evacuó el área con conocimientos certeros sobre cuáles eran las áreas de seguridad establecidas.

En Boca Sur Viejo, el 8\% de la población reaccionó ante el evento de forma inadecua$\mathrm{da}$, es decir personas que se quedaron en su casa pese al llamado de Bomberos a evacuar; un $66 \%$ de la población reaccionó de manera adecuada, es decir evacuó el área, dejó sus casas y se dirigió a un lugar que creía seguro, un $24 \%$ de la población sabia cuáles eran los lugares seguros y se dirigió a ellas. 
Figura $\mathrm{N}^{\circ} 6$

Nivel de vulnerabilidad según el tipo de vivienda e Índice de Bienestar Social (IBS)
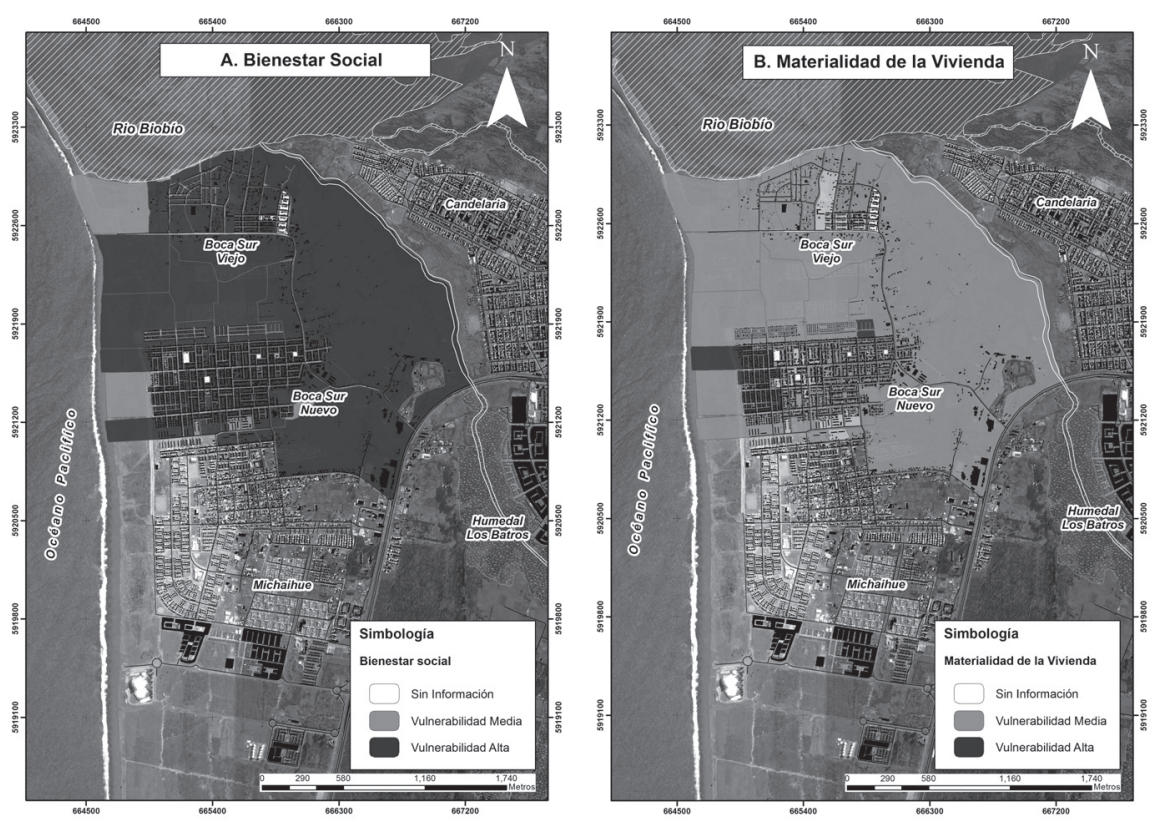

Fuente: Elaboración propia.

Figura $\mathrm{N}^{\circ} 7$

Nivel de vulnerabilidad física

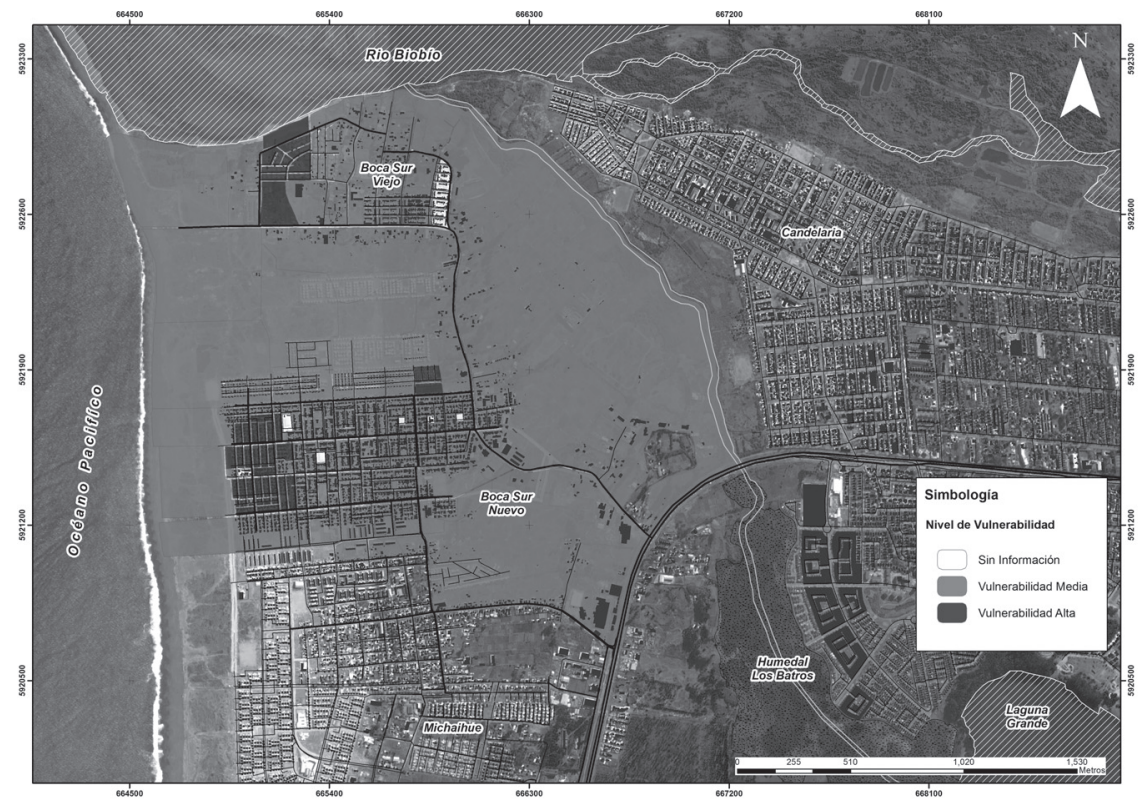

Fuente: Elaboración propia. 
RIESGO DE TSUNAMI Y PLANIFICACIÓN RESILIENTE DE LA COSTA CHILENA. LA

Figura $\mathrm{N}^{\circ} 8$

Nivel de vulnerabilidad socioeconómica

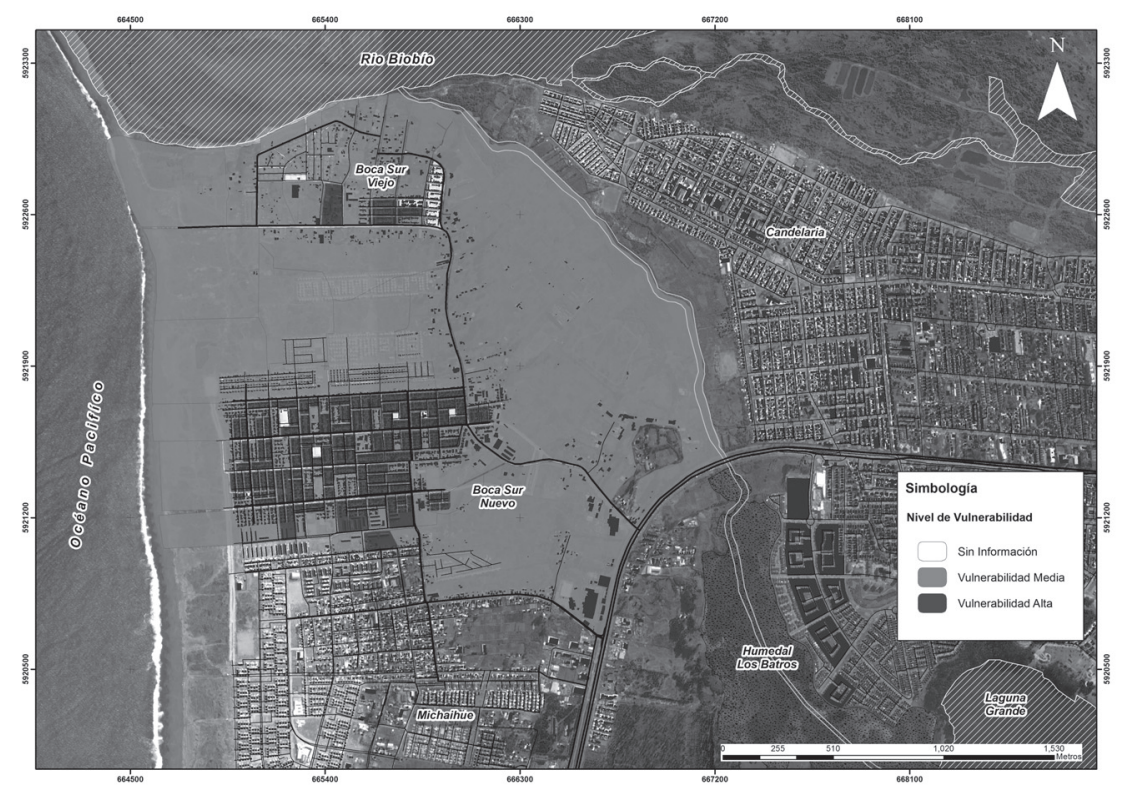

Fuente: Elaboración propia.

Figura $N^{\circ} 9$

Nivel de vulnerabilidad organizacional

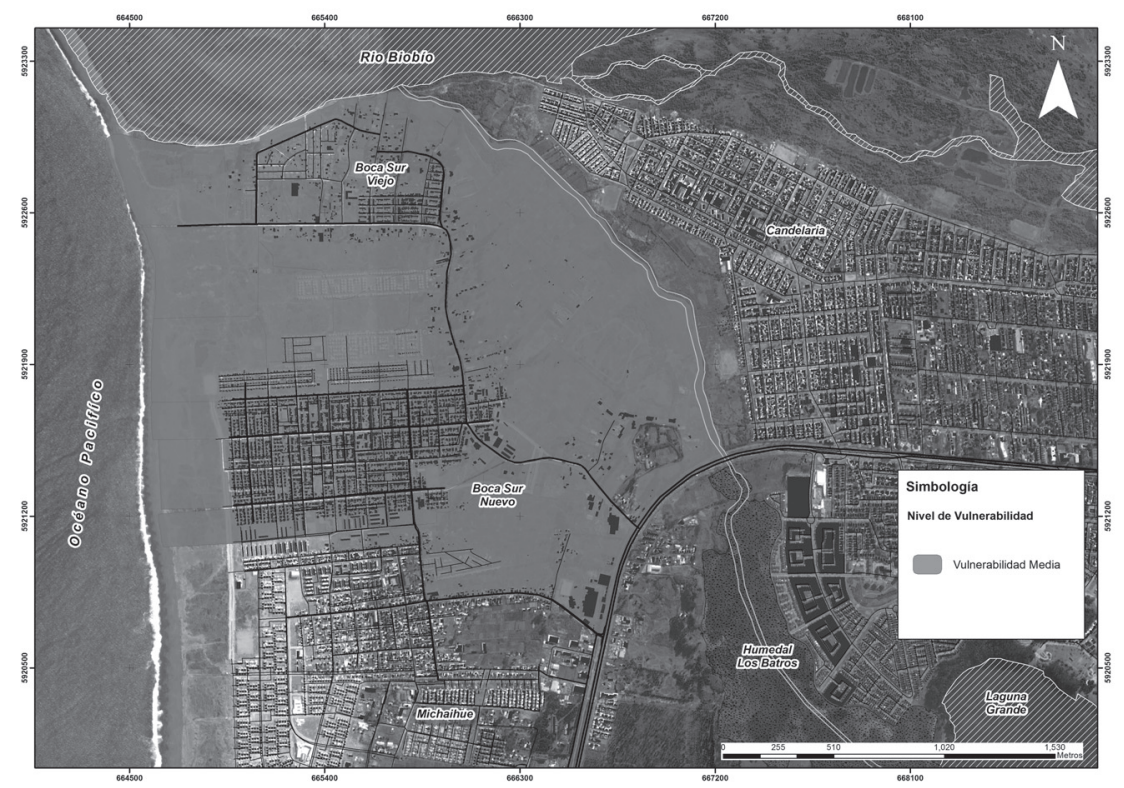

Fuente: Elaboración propia. 
Áreas de riesgo de inundación por tsunami

Las áreas de riesgo alto correspondieron a una franja del litoral que se extiende hasta unos $500 \mathrm{~m}$ de la playa, comprenden unos $0,54 \mathrm{~km}^{2}$ y alcanza la cota de $5 \mathrm{msnm}$ (Figura $N^{\circ} 10$ ). Esta franja incluye parte de la localidad de Boca Sur Viejo y las poblaciones más cercanas a la playa en Boca Sur Nuevo (entre calle Pasaje 1 y Av. Venus). El riesgo aquí está condicionado por las mayores alturas de flujo generadas por el tsunami y por factores de vulnerabilidad asociados al tipo y a la precariedad de la vivienda, así como el nivel socioeconómico de la población (IBS) y una mala organización comunitaria para la evacuación.

Las áreas con riesgo medio abarcaron un área mayor $\left(0,72 \mathrm{~km}^{2}\right)$, distribuyéndose entre la zona adyacente a la playa y al interior de la planicie litoral donde se localizan las poblaciones de Boca Sur Viejo y Nuevo, hasta la cota de los $5 \mathrm{msnm}$. Otro sector afectado, corresponde al estero Los Batros, hasta una distancia de $675 \mathrm{~m}$ de la desembocadura (5 msnm).
Las áreas de riesgo bajo $\left(3,22 \mathrm{~km}^{2}\right)$, se localizan principalmente en dos sectores principales: próxima a la playa entre Boca Sur Viejo y Nuevo, donde las alturas son mayores y existe una escasa ocupación humana y otra, ubicada al interior de la llanura y a unos $300 \mathrm{~m}$ de la calle Daniel Belmar, donde el tsunami ya no sería percibido y donde la materialidad de las construcciones tiene mejores estándares de calidad.

En la Figura $\mathrm{N}^{\circ} 10$, se ha incluido la localización de las vías de evacuación determinadas por la Municipalidad de San Pedro. De acuerdo con esto, la extensión de cada vía es de $1,3 \mathrm{~km}$ (Av. Venus) y de $1,1 \mathrm{~km}$ (Pasaje 1). Ambas deben interceptar la Av. Daniel Belmar, que es la única vía de evacuación existente hacia la Ruta 160 que une Coronel con San Pedro de la Paz, la cual tiene una extensión de 2,5 km. Asumiendo una velocidad media de escape de $1,1 \mathrm{~m} / \mathrm{s}$ (una persona caminando lento), el tiempo de evacuación necesario para los habitantes de Boca Sur Viejo en su recorrido por la Av. Daniel Belmar sería de 38 minutos. Para personas ancianas, el tiempo de evacuación aumenta a 56 minutos, asumiendo una velocidad de escape de 0,75 $\mathrm{m} / \mathrm{s}$.

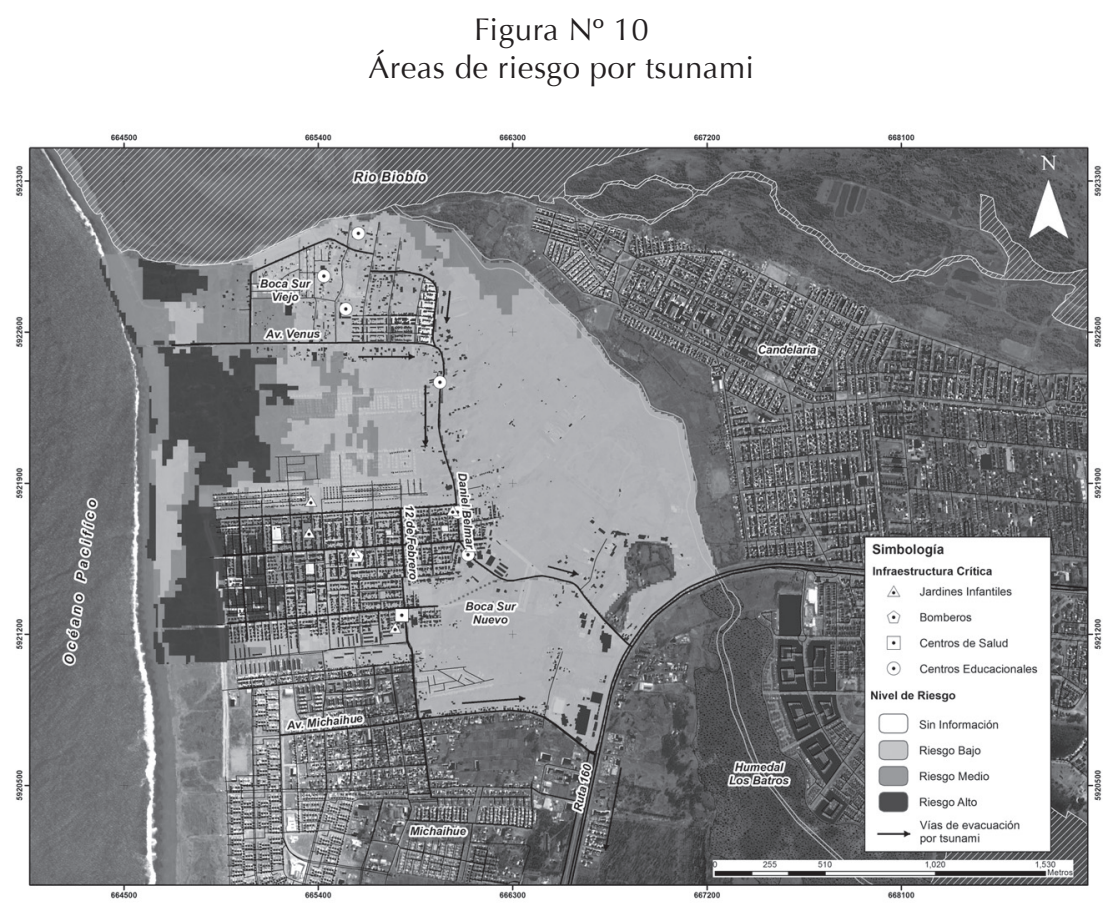

Fuente: Elaboración propia. 


\section{Análisis de los factores de vulnerabilidad de la localidad de Boca Sur}

En esta localidad, se conjugan una serie de factores que explican la situación de riesgo, donde el $28 \%$ del área presenta alguna de las categorías de riesgo alto o medio: localización inadecuada sobre una planicie de playa con geoformas costeras degradadas por la intervención humana; crecimiento urbano exponencial en las últimas décadas, en el contexto de Área Metropolitana; condiciones socioeconómicas de alta vulnerabilidad social, población poco organizada y desinformada sobre mecanismos de prevención o evacuación y una estructura urbana poco acorde a necesidades de evacuación en caso de escenarios extremos de tsunami.

Efectivamente esta localidad forma parte de una de las comunas con mayor crecimiento urbano de las últimas décadas y donde grandes proyectos inmobiliarios se han concentrado en las terrazas más altas (Lagunas Grande y Chica), sin embargo es una las áreas con mayor inequidad social (índice de Gini de 0.541, encuesta Casen 2006) y donde se ha producido históricamente mayor degradación ambiental. El estudio realizado por Vásquez y Salgado (2011) establecen una exposición a una amplia tipología de riesgos ambientales, no solo tsunami, sino también inundaciones mareales, remoción en masa, erosión eólica y problemas tales como contaminación por descargas de emisarios sanitarios (existen 3 en el área) y vertederos ilegales, estos últimos fácilmente observables en la desembocadura del río Biobío, una de las áreas húmedas más degradadas del sector.

Las condiciones socioeconómicas que presenta la población del área, la hace potencialmente más vulnerable ante un desastre de gran magnitud, al respecto el $61 \%$ de la población registró alta vulnerabilidad socioeconómica. La historia de erradicaciones masivas en los años '60 en las ciudades de Concepción y Talcahuano, posteriormente con los programas de viviendas sociales han tipificado estos asentamientos como barrios precarios y vulnerables. Según el Pladeco (2012), a través del Sistema de Protección
Social Chile Solidario-Programa Puente, de un total de 3.135 familias incorporadas al programa, 1.479 (47\%) viven en Boca Sur y Michaihue.

Los principales factores de vulnerabilidad en el área se asociaron a la precariedad de la vivienda, la alta densidad de población (viviendas de bloques), los bajos niveles de bienestar social (alto IBS) sumado a la escasa organización de la población para evacuar con conocimiento hacia cotas seguras. Al respecto, el Pladeco (2012) se refiere al precario capital social y cultural en estas localidades, difícil de erradicar a pesar de la inversión social realizada y considerando que se trata de una comuna joven. Por otro lado, esto contrasta con otros sectores de la comuna que han sido beneficiadas con una inversión notoria especialmente en la creación de barrios residenciales de altos ingresos, dotados de infraestructura vial y áreas verdes, produciéndose así un desarrollo económico desigual y de fuerte inequidad social, favoreciendo la segregación social. Esto último se verifica al observar el número de familias inscritas o que reciben beneficios del programa Chile Solidario, es decir unas 2.292 familias, todas ellas residentes de Boca Sur, Candelaria y Michaihue.

A su vez, conocer los tiempos de arribo de las primeras ondas de un tsunami, se transforma en una herramienta vital al momento de manejar la emergencia y dar la alerta temprana ante eventos. El caso de Boca Sur, el ingreso del primer tren de ondas es 22 minutos luego de ocurrido el terremoto y la población más cercana a la playa está aproximadamente a $2 \mathrm{~km}$ de distancia de la zona de seguridad (ruta 160). De este modo, la población de Boca Sur Viejo sería la más afectada debido al rango etario y perfil socieconómico, los cuales requerirían aproximadamente entre 40 minutos y 1 hora para llegar a zona segura (a pie), dependiendo del rango etario y las condiciones de discapacidad. Considerando el diseño de la red vial y las dificultades de trayectoria lineal hacia la Av. Daniel Belmar (vía férrea), se presentaría una alta posibilidad que los evacuados no alcancen la zona segura antes de la llegada de la primera onda, situación que se repite en otras ciudades del país, por ejemplo en La Serena (Tamburini, 2014) e Iquique (Urra et 
al., 2015). De ahí que el diseño vial deba ser pensado conjuntamente con el diseño de vías de evacuación en una planificación territorial integrada a la gestión del riesgo con el fin de reducir la vulnerabilidad.

Es importante destacar que se ha investigado el efecto de las geoformas submarinas tales como cañones submarinos, sobre la propagación e impacto de los tsunamis y se ha establecido que estos pueden ser capaces de modificar la amplitud del tsunami, con resultados contradictorios, es decir a veces amplificándola o reduciéndola (loualalen et al., 2007; Divyalakshmi et al., 2011; Iglesias et al., 2014). Por otro lado, son capaces de modificar la velocidad de arribo de los trenes de ondas y generar ondas de orilla (Iglesias et al., 2014). En el caso, del área de estudio, Aránguiz \& Shibayama (2013) demostraron que la presencia del cañón submarino del Biobío juega un rol fundamental en la propagación de tsunami en el golfo de Arauco, de tal modo que las ondas del tsunami se refractan debido al cañón submarino y luego de difractan debido a la presencia de la isla Santa María, lo que hace que los frentes de ondas viajen prácticamente de norte a sur sin impactar directamente la costa de San Pedro de La Paz. Por lo tanto, el comportamiento descrito anteriormente, sumado a que el tsunami de 2010 ocurrió en marea baja, es responsable de que no hubiera inundación significativa en la zona en estudio, lo cual no implica que el área no esté ajena a la amenaza de tsunami, considerando otros escenarios.

De la misma forma que las geoformas submarinas modifican los trenes de ondas y su incidencia en la costa, las geoformas y los ecosistemas costeros juegan un rol relevante en la mitigación de los impactos de un tsunami, estos ya han sido demostrados en casos como Japón, Sri Lanka e Indonesia, especialmente a través del rol de humedales costeros (Chen, et al., 2005; Danielsen et al., 2005: Tanaka et al., 2007; Chatenoux \& Peduzzi, 2007). Playas, dunas y humedales atenúan la velocidad y altura de inundación y por lo tanto su poder destructivo, situación que en el área de estudio presentan alta degradación, en especial dada la tendencia erosiva de la costa de Escuadrón (Martínez, 2013a).

Aquí, el entorno natural y paisajístico de Boca Sur se encuentra altamente degradado
(Smith y Romero, 2009; Vásquez y Salgado, 2011; Rojas et al., 2015), existe urgencia en recuperarlos por su función estabilizadora del litoral y otras tales como: servir de mecanismo de disipación natural frente a amenazas naturales, tales como tsunamis, marejadas y crecidas de ríos; servir de patrimonio natural para desarrollar economías locales; servir de reservorios, aportar servicios ecosistémicos a la comuna y servir de lugares de restauración en situaciones de postdesastre. Desde el punto de vista del manejo de emergencias y recuperación postdesastre, estos sectores suelen convertirse en lugares restauradores desde un punto de vista físico y cultural, ya que los mismos habitantes utilizan para proveer de servicios básicos (agua) e inclusive restauración emocional, tal como fue probado con el terremoto de 1960 en Valdivia y 2010 en San Pedro (Villagra y Rojas, 2013; Villagra \& Dobbie, 2014; Villagra \& Felsenhardt, 2015).

\section{Conclusiones y recomendaciones}

El $28 \%$ de la localidad presentó riesgo alto o medio, situación que se explica principalmente por un factor de localización geográfica donde una extensa planicie de playa ha sido urbanizada de manera creciente generando una importante exposición de vidas humanas y de infraestructura crítica al riesgo de tsunami, con ondas que pueden llegar a la costa luego de 22 minutos de ocurrido el terremoto y que pueden alcanzar la cota de 5 msnm y alturas de flujo de $2 \mathrm{~m}$. Los factores de vulnerabilidad que explican el riesgo se asociaron a una alta precariedad de la vivienda, un bajo nivel de bienestar social y niveles de ingreso, una alta densidad poblacional y un bajo nivel de organización comunitaria de la población frente a mecanismos de evacuación.

Esta condición genera una fuerte necesidad de incorporar elementos aplicables a la planificación para la resiliencia de la costa como vía para reducir el impacto potencial de los desastres, como el que ocurrió en esta costa en 2010, la cual opera sobre la base del desarrollo de capacidades de amortiguación, autoorganización social y aprendizaje. En este sentido, la planificación territorial debe reconsiderar este tipo de áreas, como lugar 
de expansión urbana como han sido tratadas hasta ahora o como lugar de localización de viviendas sociales, debido a que ello aumenta las condiciones de vulnerabilidad de la población. Se requiere aquí pensar en un modelo de asentamiento de baja densidad en las áreas próximas a la línea litoral con una red vial que cumpla la doble función de sustentar un potencial de evacuación y a la vez un transporte urbano y priorizar el equipamiento comunitario como forma de fortalecer a su vez la organización social y elevar la participación comunitaria en torno a situaciones de emergencia, articulados a través de programas de prevención continuos y formación de actores sociales claves. Especialmente es relevante la recuperación de la identidad territorial (en este caso menoscabada por un proceso de erradicación de campamentos) y la falta de valoración por el entorno natural, agravadas por el desarrollo de actividades antrópicas de alto impacto que han generado una pérdida importante del patrimonio natural de la costa, principalmente degradación y reducción de la superficie de campos dunares y humedales, los principales ambientes costeros prestadores de servicios ambientales y capaces de mitigar los efectos de los tsunamis. Estos ambientes se están perdiendo aquí, y en una buena parte del país lo cual conjugado a un intenso proceso de crecimiento urbano en la costa, explican el aumento de la recurrencia y la magnitud de los desastres naturales en Chile en los últimos años.

\section{Referencias bibliográficas}

ADGER, W. Vulnerability. Global Environmental Change, 2006, Vol. 16, № 3, p. 268-281.

ANGELL, E. \& STOKKE, K. Vulnerability and adaptive capacity in Hammerfest, Norway. Ocean \& Coastal Management, 2014, No 94 , p. 56-65.

ARÁNGUIZ, R. Modelación numérica del tsunami de 2010 en la Bahía de Concepción utilizando deformación inicial estática no uniforme. Obras y Proyectos, 2010, № 8, p. 12-18.

ARÁNGUIZ, R. \& SHIBAYAMA, T. The effect of submarine canyon on tsunami pro- pagation: A case study of the Biobio Canyon, Chile. Coastal Engineering Journal, 2013, Vol. 55, $\mathrm{N}^{\circ} 4,1350016$.

ARÁNGUIZ, R.; SHIBAYAMA, T. \& YAMAZAKI, Y. Tsunamis from the Arica-Tocopilla source región and their effects on port of Central Chile. Natural Hazards, 2014, No 71, p. 175-202.

ARÁNGUIZ, R.; GONZÁLEZ, G.; GONZÁLEZ, J.; CATALÁN, P.; CIENFUEGOS, R.; YAGI, Y.; OKUWAKI, R.; URRA, L.; CONTRERAS, L.; DEL RIO, I. \& ROJAS, C. The 16 September 2015 Chile Tsunami from the PostTsunami Survey and Numerical Modeling Perspectives. Pure and Applied Geophysics, 2015, Vol. 173, No 2, p. 333-348.

BAERISWYL, S. Crecimiento urbano del Gran Concepción. Patrones y tendencias de una metropolización. En: HIDALGO, R.; DE MATTOS, C. y ARENAS, F. (editores) Del país urbano al país metropolitano. Santiago de Chile: Serie GEOlibros, Instituto de Geografía, Pontificia Universidad Católica de Chile, 2009, p. 241-255.

BLAIKIE, P.; CANNON, T.; DAVIES, I. \& WISNER, B. At Risk. Natural Hazards, People's Vulnerability and Disasters. New York: Routledge, 1994.

CHATENOUX, B. \& PEDUZZI, P. Impacts from the 2004 Indian Ocean Tsunami: analysing the potential protecting role of environmental features. Natural Hazards, 2007, Vol. $40, N^{\circ} 2$, p. 289-304.

CHEN, P.; LIEW, S. \& KWOH, L. Tsunami damage assessment using high-resolution satellite imagery: a case study of Aceh, Indonesia. Geoscience and Remote Sensing Symposium, 2005, Vol. 2, p. 1405-1408.

COCHARD R.; RANAMUKHAARACHCHI, S.; SHIVAKOTI, G.; SHIPIN, O.; EDWARDS, P. \& SEELAND, K. The 2004 tsunami in Aceh and Southern Thailand: A review on coastal ecosystems, wave hazards and vulnerability. Perspectives in Plant Ecology, Evolution and Systematics, 2008, Vol. 10, N ${ }^{\circ}$ 1, p. 3-40. 
CUTTER, S. Vulnerability to environmental hazards. Progress in Human Geography, 1996, Vol. 20, No 4, p. 529-539.

CUTTER, S.; BORUFF, B. \& SHIRLEY, W. Social vulnerability to environmental hazards. Social Science Quarterly, 2003, Vol. 84, № 2, p. 242-261.

CUTTER, S.; BARNES, L.; BERRY, M.; BURTON, C.; EVANS, E.; TATE, E. \& WEBB, J. A place-based model for understanding community resilience to natural disasters. Global Environmental Change, 2008, Vol. 18, № 4, p. 598-606.

DANIELSEN, F.; SØRENSEN, M.; OLWIG, M.; SELVAM, V.; PARISH, F.; BURGESS, N.; HIRAISHI, T.; KARUNAGARAN, V.; RASMUSSEN, M.; HANSEN, L.; QUARTO, A. \& SURYADIPUTRA, N. The Asian tsunami: a protective role for vegetation. Science, 2005, Vol. 310, N 5748, p. 643.

DURA, T.; CISTERNAS, M.; HORTON, B.; ELY, L.; WESSON, R.; PILARCZYK, J. \& NELSON, A. Coastal evidence for Holocene subduction-zone earthquakes and tsunamis in central Chile. Quaternary Science Reviews, 2015, Vol. 113, p. 93-111.

ECKERT, S.; JELINEK, R.; ZEUG, G. \& KRAUSMANN, E. Remote sensing-based assessment of tsunami vulnerability and risk in Alexandria, Egypt. Applied Geography, 2012, Vol. 32, № 2, p. 714-723.

GALLOPIN, G. Linkages between vulnerability, resilience, and adaptive capacity. Global Environmental Change, 2006, Vol. 16, No 3, p. 293-303.

GARRETT, E.; SHENNAN, I.; WOODROFFE, S.; CISTERNAS, M.; HOCKING, E. \& GULLIVER, P. Reconstructing paleoseismic deformation, 2: 1000 years of great earthquakes at Chucalén, south central Chile. Quaternary Science Reviews, 2015, № 113, p. 112-122.

GEIST, E. \& PARSONS, T. Probabilistic analysis of tsunami hazards. Natural Hazards, 2006, Vol. 37, No3, p. 277-314.
GREZIO, A.; GASPARINI, P.; MARZOCCHI, W.; PATERA, A. \& TINTI, S. Tsunami risk assessments in Messina, Sicily - Italy. Natural Hazards Earth System Science, 2012, Vol. 12, № 1, p. 151-163.

GOFF, J.; TERRY, J.; CHAGUÉ-GOFF, C.GOTO, K. What is a mega-tsunami? Marine Geology, 2014, Vol. 358, p. 12-17.

GRILLI, S.; O'REILLY, C.; HARRIS, J.; BAKHSH, T.; TEHRANIRAD, B.; BANIHASHEMI, S.; KIRBY, T.; BAXTER, C.; EGGELING, T.; MA, G. \& SHI, F. Modeling of SMF tsunami hazard along the upper US East Coast: detailed impact around Ocean City, MD. Natural Hazards, 2015, Vol. 76, N 1, p. 705-746.

IGLESIAS, O.; LASTRAS, G.; SOUTO, C.; COSTA, S. \& CANALS, M. Effects of coastal submarine canyons on tsunami propagation and impact. Marine Geology, 2014, Vol. 350, p. 39-51.

ILABACA, P. Evolución de la costa de Concepción: el caso de las bahías de Concepción y San Vicente. Biología Pesquera, $1989, \mathrm{~N}^{\circ} 18$, p. 29-35.

INSTITUTO NACIONAL DE ESTADÍSTICAS (INE). Chile. Ciudades, pueblos y aldeas. Censo 1992. Santiago de Chile: INE, 1992.

INSTITUTO NACIONAL DE ESTADÍSTICAS (INE). Censos de Población y Vivienda año 2002. Región del Bio-Bío. Santiago de Chile: INE, 2003.

ISLA, F.; QUEZADA, J.; MARTÍNEZ, C.; FERNÁNDEZ, A. \& JAQUE, E. The evolution of the Bío-Bío delta and the coastal plains of the Arauco Gulf, Bio-Bío Region: the Holocene sea-level curve of Chile. Journal of Coastal Research, 2012, Vol. 28, № 1, p. 102-111.

IOUALALEN, M.; PELINOVSKY, E.; ASAVANANT, J.; LIPIKORN, R. \& DESCAMPS, A. On the weak impact of the 26 December Indian Ocean tsunami on the Bangladesh coast. Natural Hazards and Earth System Sciences, 2007, Vol. 7, № 1, p. 141-147.

JELÍNEK, R.; KRAUSMANN, E.; GONZÁLEZ, M.; ÁLVAREZ-GÓMEZ, J.; BIRKMANN, J. \& WELLE, T. Approaches for tsuna- 
mi risk assessment and application to the city of Cádiz, Spain. Natural Hazards, 2011, Vol. $60, \mathrm{~N}^{\circ} 2$, p. 273-293.

JIN, D. \& LIN, J. Managing tsunamis through early warning systems: A multidisciplinary approach. Ocean \& Coastal Management, 2011, Vol. 54, N 12, p. 189-199.

KHEW, Y.; JARZEBSKI, M.; DYAH, F.; SAN CARLOS, R.; GU, J.; ESTEBAN, M.; ARÁNGUIZ, R. \& AKIYAMA, T. Assessment of social perception on the contribution of hard-infrastructure for tsunami mitigation to coastal community resilience after the 2010 tsunami: Greater Concepcion area, Chile. International Journal of Disaster Risk Reduction, 2015, Vol. $13, N^{\circ} 1$, p. 324-333.

KOX, S.; VILA, R.; KEULERS, B.; KOUDSTAAL, K.; CRIELAARD, R. \& VEERMAN, M. Multi-criteria approach to the infrastructural problem of San Pedro de la Paz: from an urbanistic and hydraulic perspective. Multidisciplinary Project, Delft University of Technology, 2015. Disponible en Internet: http://repository.tudelft.nl/view/ ir/uuid\%3 A 36 df 7d 7 5-8a 83 - 4db6-8bd508f728e6089f/

KULIKOV, E.; RABINOVICH, A. \& THOMSON, R., 2005. Estimation of tsunami risk for the coasts of Peru and Northern Chile. Natural Hazards, 2005, Vol. 35, N², p. 185-209.

LAGOS, M. Tsunamis de origen cercano a las costas de Chile. Revista de Geografía Norte Grande, 2000, № 27, p. 93-102.

LAGOS, M. y GUTIÉRREZ, D. Simulación del tsunami de 1960 en un estuario del centro-sur de Chile. Revista de Geografía Norte Grande, 2005, № 33, p. 5-18.

LAGOS, M. y CISTERNAS, M. El nuevo riesgo de tsunami: considerando el peor escenario. Revista Scripta Nova, 2008, Vol. XII, $N^{\circ}$ 270. Disponible en Internet: http://www. ub.edu/geocrit/sn/sn-270/sn-270-29.htm

LEÓN, J. \& MARCH, A. Urban morphology as a tool for supporting tsunami rapid resilience: A case study of Talcahuano, Chile. Habitat International, 2014, No 43, p. 250-262.
LOCKRIDGE, P. Tsunamis in Chile-Perú. Boulder: Report SE-39 Worl Data Centera for solid earth and geophysics 1985 .

LØVHOLT, F.; SETIADI, N.; BIRKMANN, J.; HARBITZ, C.; BACH, C.; FERNANDO, N.; KAISER, G. \& NADIM, F. Tsunami risk reduction - are we better prepared today than in 2004? International Journal of Disaster Risk Reduction, 2014, Vol. 10, p. 127-142.

LØVHOLT, F.; GLIMSDAL, S.; HARBITZ, C.; HORSPOOL, N.; SMEBYE, H.; DE BONO, A. \& NADIM, F. Global tsunami hazard and exposure due to large co-seismic slip. International Journal of Disaster Risk Reduction, 2014, Vol. 10, p. 406-418.

MARTÍNEZ, C. Vulnerabilidades y desastres socio-naturales: aportes desde la Geografía al proceso de Reconstrucción en la Región del Biobío. Revista Geográfica del Sur, 2013, Vol. 3, No 2, p. 33-58.

MARTÍNEZ, C. Evolución costera, procesos morfodinámicos posterremoto y manejo costero en la región del Biobío, Chile. In: XXXIV Congreso Nacional y XVIII Internacional de Geografía, Universidad del Biobío, Chillán, 15 al 19 octubre de 2013a, p.4.

MARTÍNEZ, C.; ROJAS, O.; ARÁNGUIZ, R.; BELMONTE, A.; QUEZADA, J.; ALTAMIRANO, A. y FLORES, P. Riesgo de tsunami en Caleta Tubul, Región del Biobío: escenarios extremos y transformaciones territoriales posterremoto. Revista de Geografía Norte Grande, 2012, No 53, p. 85-106.

MARTÍNEZ, C. Factores de vulnerabilidad y Reconstrucción posterremoto en tres localidades costeras chilenas: ¿generación de nuevas áreas de riesgo? Bulletin de I'Institut Français d'Études Andines, 2014, Vol. 43, No 3, p. 529-558.

MAS, E.; KOSHIMURA, S.; SUPPASRI, A.; MATSUOKA, M.; MATSUYAMA, M.; YOSHII, T.; JIMENEZ, C.; IMAMURA, F. \& YAMAZAKI, F. Developing Tsunami fragility curves using remote sensing and survey data of the 2010 Chilean Tsunami in Dichato. Natural Hazards Earth System Science, 2012, No 12, p. 2689 2697. 
MINISTERIO DE PLANIFICACIÓN Y COPERACIÓN (MIDEPLAN). Encuesta de Caracterización Socio-Económica Nacional (CASEN) 2003 y 2006. Santiago de Chile: MINVU, 2002. Disponible en Internet: http://www.observatoriourbano.cl/index.asp

MINISTERIO DE OBRAS PÚBLICAS (MOP). Guía Práctica para la Vivienda de Emergencia. Santiago de Chile: Dirección de Arquitectura, 2014.

MINISTRY OF LAND INFRASTRUCTURE, TRANSPORT AND TOURISM. Guide to Determining the Potential Tsunami Inundation (Temporary Translation). Tokyo: Seacoast Office, Water and Disaster Management Bureau, and Coast Division, River Department, National Institute for Land and Infrastructure Management, Ministry of Land, Infrastructure, Transport and Tourism. Ver 2. October 2012.

MUNICIPALIDAD DE SAN PEDRO DE LA PAZ. Plan de Desarrollo Comunal. Comuna de San Pedro de La Paz, 2012- 2016. Disponible en Internet: http://sanpedrodelapaz. $\mathrm{cl} / \mathrm{wp}$-content/uploads/2013/10/pladeCo_2012_2016.pdf

OKEY, T.; AGBAYANI, S. \& ALIDINA, H. Mapping ecological vulnerability to recent climate change in Canada's Pacific marine ecosystems. Ocean \& Coastal Management, 2015, Vol. 106, p. 35-48.

PALACIOS, A. Sismicidad histórica de la ciudad de Concepción desde su fundación en 1550 hasta su traslado en 1751. Santiago de Chile: Servicio Nacional de Geología y Minería, Boletín № 64, 2012.

PAPAZACHOS, B.C.; SCORDILIS, EM.; PANAGIOTOPOULOS, DG.; PAPAZACHOS, CB. \& KARAKAISIS, GF. Global relations between seismic fault parameters and moment magnitude of Earthquakes. Bulletin of the Geological Society of Greece, 2004, Vol. XXXVI, p. 1482-1489.

PAYANDE, A.; NIKSOKHAN, M. \& NASERIAN, H. Tsunami hazard assessment of Chabahar bay related to megathrust seismogenic potential of the Makran subduction zone. Natural Hazards Earth System Science, 2015, Vol. 76, p. 161-176.
PRERNA, R.; KUMAR, T.; MAHENDRA, R. \& MOHANTY, P. Assessment of Tsunami Hazard Vulnerability along the coastal environs of Andaman Islands. Natural Hazards, 2015, Vol. 5, p. 701-726.

RATNA, S. A Gis based approach of an evacuation model for tsunami risk reduction. Journal of Integrated Disaster Risk Management, 2012, Vol. 2, № 2, p. 108-139.

RATNA, S.; NIENDYAWATI, S. \& SUWAHYUONO, S. Remote Sensing for disaster mitigation: Case Study for tsunami evacuation route modelling in Cilacap-Central Java, Indonesia. In: International Archives of the Photogrammetry, Remote Sensing and Spatial Information Science, 2010, Vol. XXXVIII, N ${ }^{\circ}$ 8, p. 281-286.

RHODES, B.; KIRBY, M.; JANKAEW, K. \& CHOOWONG, M. Evidence for a mid-Holocene tsunami deposit along the Andaman coast of Thailand preserved in a mangrove environment. Marine Geology, 2011, Vol. 282, №3-4, p. 255-267.

ROJAS, O.; SÁEZ, K.; MARTÍNEZ, C. y JAQUE, E. Efectos ambientales postcatástrofe en localidades costeras afectadas por tsunami en Chile: desde la emergencia a la Reconstrucción. Interciencia, 2014, Vol. 39, № 6, p. 383-390.

ROJAS, O. y MARTÍNEZ, C. Riesgos Naturales: evolución y modelos conceptuales. Revista Universitaria de Geografía, 2011, № 20, p. 83-116.

ROJAS, C.; SEPÚLVEDA, E.; BARBOSA, O.; ROJAS, O. y MARTíNEZ, C. Patrones de Urbanización y Biodiversidad en Humedales Urbanos en Concepción Metropolitano. Revista de Geografía Norte Grande, 2015, No 61, p. 181-204

ROJAS, C.; MUÑIZ, I. \& PINO, J. Understanding the Urban Sprawl in the Mid-Size Latin American Cities Through the Urban Form: Analysis of the Concepcion Metropolitan Area (Chile). Journal of Geographic Information System, 2013, Vol. 5, No 3, p. 222-234. 
SHAW, D.; SCULLY, J. \& HART, T. The paradox of social resilience: How cognitive strategies and coping mechanisms attenuate and accentuate resilience. Global Environmental Change, 2014, Vol. 25, No 1, p. 194-203.

SHIBAYAMA, T.; ESTEBAN, M.; NISTOR, I.; TAKAGI, H.; THAO, N.; D., MATSUMARU, R.; MIKAMI, T.; ARANGUIZ, R.; JAYARATNE, R. \& OHIRA, K. Classification of Tsunami and Evacuation Areas. Natural Hazards, 2013, Vol. 67, No 2, p. 365-386.

SMITH, P. y ROMERO, H. Efectos del crecimiento urbano del Área Metropolitana de Concepción sobre los humedales de Rocuant-Andalién, Los Batros y Lenga. Revista de Geografía Norte Grande, 2009, № 43, p. 81-93.

SUGAWARA, D. \& GOTO, K. Numerical modeling of the 2011 Tohoku-oki tsunami in the offshore and onshore of Sendai Plain, Japan. Sedimentary Geology, 2012, Vol. 282, $\mathrm{N}^{\circ} 1-2$, p. 110-123.

SUPPASRI, A.; KOSHIMURA, S. \& IMAMURA, F. Developing tsunami fragility curves based on the satellite remote sensing and the numerical modeling of the 2004 Indian Ocean tsunami in Thailand. Natural Hazards Earth System Science, 2001 Vol. 11, $N^{\circ} 1$, p. 173-189.

TAMBURINI, L. Evaluación del potencial de evacuación frente a la amenaza de tsunami en la zona costera de la ciudad de La Serena, Región de Coquimbo. Santiago de Chile: Tesis para optar al Grado de Magíster, Instituto de Geografía, Pontificia Universidad Católica de Chile, 2014.

TANAKA, N.; SASAKI, Y.; MOWJOOD, M.; JINADASA, K. \& HOMCHUEN, S. Coastal vegetation structures and their functions in tsunami protection: experience of the recent Indian Ocean tsunami. Landscape Ecology Engineering, 2004, № 3, p. 33-45.

TURNER I.; KASPERSON, R.; MATSON, P.; MCCARTHY, J.; CORELL, R.; CHRISTENSEN, L.; ECKLEY, N.; KASPERSON, J.; LUERS, A.; MARTELLO, M.; POLSKY, C.; PULSIPHER, A. \& SCHILLER, A. Framework for vulnerability analysis in sustainability sci- ence. In: Proceedings of the National Academy of Sciences of the United States of America, 2003, Vol. 100, p. 8074-8079.

URRA, L.; MAS, E.; ARÁNGUIZ, R.; ADRIANO, B. \& KOSHIMURA, S. Estimación de daño por tsunami en Iquique utilizando modelación numérica y curvas de fragilidad. En: XXII Congreso Chileno de Ingeniería Hidráulica, Santiago de Chile, octubre de 2015.

VÁSQUEZ, A. y SALGADO, M. Desigualdades socioeconómicas y distribución inequitativa de los riesgos ambientales en las comunas de Peñalolén y San Pedro de la Paz: Una perspectiva de justicia ambiental. Revista de Geografía Norte Grande, 2009, № 43, p. 95-110.

VILLAGRA, P. \& FELSENHARDT, C. Emergency urban landscapes in Valdivia Chile: Contributions to the post-disaster planning and urban design processes within a restoration context. Revista INVI, 2015, No 30, p. 19-78.

VILLAGRA, P. \& DOBBIE, M. Design aspects of urban wetlands in an earthquakeprone environment. Journal of Urban Design, 2014, Vol. 19, № 5, p. 660-681.

VILLAGRA, P. y ROJAS. C. Dimensiones física y cultural de la resiliencia postdesastre: ¿con compatibles en ciudades chilenas? Revista Geográfica del Sur, 2013, Vol. 4, № 6, p. 85-102.

WILCHES-CHAUX, G. La vulnerabilidad global. En: MASKREY, A. (compilador). Los Desastres No Son Naturales. La Red, 1993. Disponible en Internet: http://www.desenredando.org/public/libros/1993/ldnsn/

WISNER, B.; BLAIKIE, P.; CANNON, T. \& DAVIS, I. At Risk: Natural Hazards, People's Vulnerability and Disasters. New York: Routledge, 2004.

WALSH, T.; TITOV, V.; VENTURATO, A.; MOFJELD, H. \& GONZÁLEZ, F. Tsunami Hazard Map of the Anacortes -Whidbey Island Area. Washington: Division of Geology and Earth Resources and NOAA TIME Cen- 
ter PMEL. Open File Report 2005-1, scale 1:62.500, 2005.

YAMAZAKI, Y.; KOWALIK, Z. \& CHEUNG, K. Depth-integrated, non-hydrostatic model for wave breaking and runup. International Journal for Numerical Methods in Fluids, 2009, Vol. 61, № 5, p. 473-497.

YAMAZAKI, Y.; CHEUNG, K.F. \& KOWALIK, Z. Depth-integrated, non-hydrostatic model with grid nesting for tsunami generation, propagation, and run-up. International Journal for Numerical Methods in Fluids, 2011, Vol. $67, \mathrm{~N}^{\circ} 12$, p. 2081-2107.

YANG, S.; HE, S.; DU, J. \& SUN, X. Screening of social vulnerability to natural hazards in China. Natural Hazards, 2015, No 76, p. 1-18.

YOO, G.; KIM, A. \& HADI, S. A methodology to assess environmental vulnerability in a coastal city: Application to Jakarta, Indonesia. Ocean \& Coastal Management, 2014, Vol. 102, p. 169-177. 